\title{
OBSERVATION OF SOLAR SYSTEM BODIES ON BOARD THE FUTURE RUSSIAN ASTROMETRIC SATELLITE
}

\author{
V.N.YERSHOV \\ Pulkovo Observatory, \\ 196140 St Petersburg, Russia \\ e-mail: yersh@gao.spb.su
}

\begin{abstract}
A future Russian astrometric satellite (Struve) is aimed at an extension of the fundamental coordinate system down to 18-th magnitude stars. The positional accuracy of observations is planned to be about 0.6 mas. Many of the Solar System objects will be observed by the Struve satellite. Problems of registration and reduction of these observations are discussed.
\end{abstract}

\section{Introduction}

The future Russian astrometric satellite Struve (Yershov, Kanayev 1994) will be able to detect many of asteroids, comets, planets and their satellites as well as more than 4 million program stars. The limiting magnitude of two on-board telescopes is estimated to be $V=18$ and positional accuracy 0.6 mas of observations is expected. A $40-\mathrm{cm}$ catadioptric Schmidt telescope scheme ( $2.5 \mathrm{~m}$ focal length) is chosen for the Struve satellite. Charge coupled devices are considered as the basic detectors for the focal plain assembly. Microchannel plates are also under consideration which are more suitable for detection of moving objects such as close asteroids or space debris.

\section{Scheme of observations}

The Struve satellite will produce observations in a scanning mode inertially rotating with a frequency of about 10 turns per day. Four viewing directions are organized by the use of two beam-combiners placed in front of the

S. Ferraz-Mello et al. (eds.), Dynamics, Ephemerides and Astrometry of the Solar System, 415-418. (C) 1996 IAU. Printed in the Netherlands. 


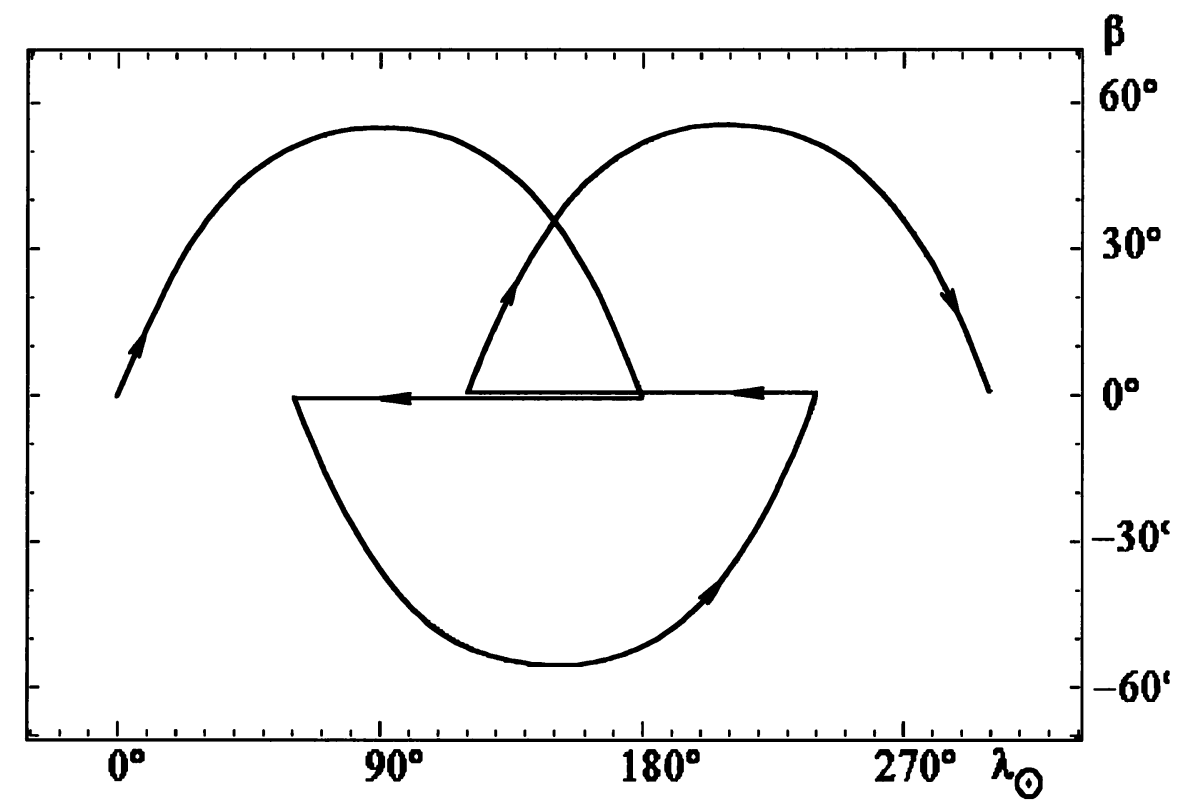

Figure 1. The Vlasov scanning law. After completing half of the current great circle the spin axis is to be switched back to the previous trajectory great circle. The aspect angle varies from $55^{\circ}$ to $60^{\circ}$, and the spin axis passes three great circles each year.

entrance pupils of two on-board telescopes. $62^{\circ}$ and $74^{\circ}$ basic angles have been chosen for the measurements. Two scanning laws are under consideration: the Hipparcos-like rotational scanning with $55^{\circ}$ aspect angle and the Vlasov scanning mode, when the spin axis moves along three intersected orthogonal great circles from one intersection point to another (Fig.1).

Solar System objects at their oppositions are not available for observations but all planets except Mercury are visible within elongations $\pm 35^{\circ}-$ $145^{\circ}$.

As most Solar System objects concentrate close to the ecliptic, it is interesting to analyze the distribution of scans there. Fig.2 and Fig.3 show this distribution for the Vlasov and the Hipparcos-like scanning modes, respectively. The Vlasov scanning mode provides more uniform coverage of the ecliptic zone.

\section{Detection}

Computer simulations of CCD detection of bright planets show relatively poor accuracy (for example, a single transit of Mars gives $50-80$ mas). 


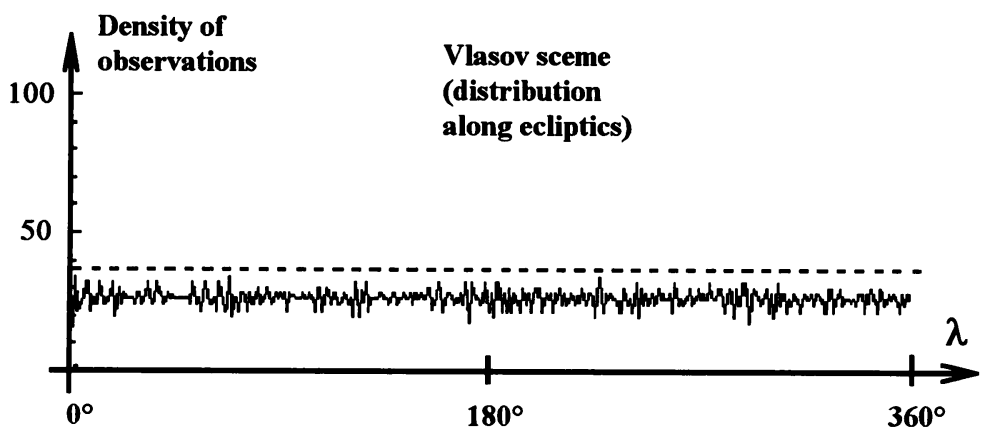

Figure 2. Distribution of density of observations along the ecliptic for the Vlasov scanning law (one year of observations).

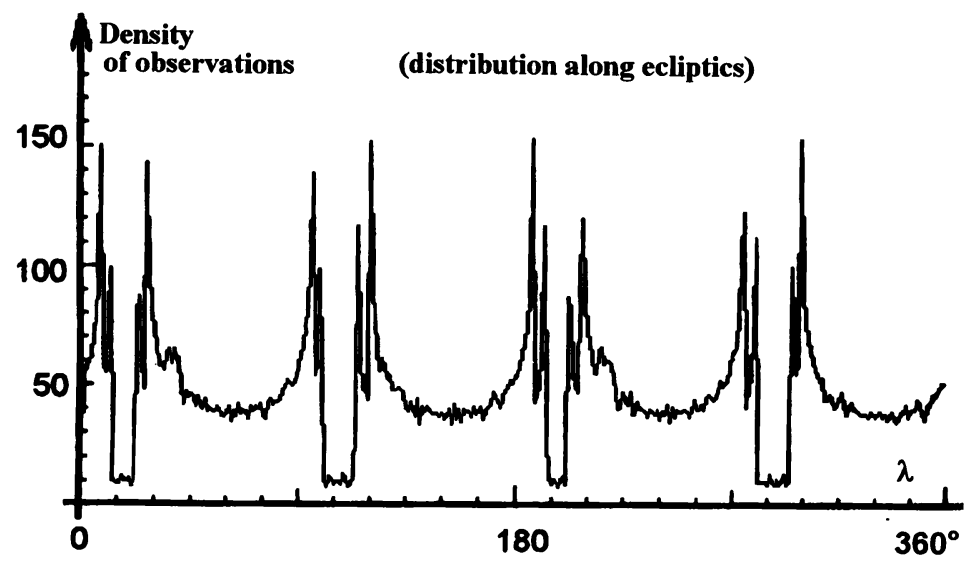

Figure 3. Distribution of density of observations along the ecliptic for the rotational scanning law (one year of observations).

Much better accuracy one may achieve by observing natural satellites of the planets. Practically, all main satellites of major planets are available for observation (except Charon, Pluto's satellite). Table 1 shows the accuracy of a single observation (in milliarcseconds) for different Solar System objects.

\section{Identification of objects}

Four observations during one scan are separated by time intervals $29.6^{m}$, $42.4^{m}, 24.8^{m}$, and $47.2^{m}$ (corresponding to the viewing directions). Each object is available for observations within three consecutive scans.

The Solar System objects move noticeably even within a single tran- 
TABLE 1. Accuracy of observations of Solar System objects (a single observation, $5^{S}$ integration time).

\begin{tabular}{lc}
\hline Object (magnitude, $V)$ & $\sigma_{1}$, mas \\
\hline Pluto $\left(15^{m}\right)$ & 11 \\
$10-\mathrm{km}$ asteroid $\left(16^{m}\right)$ & 13 \\
Triton $\left(17^{m}\right)$ & 41 \\
$1-\mathrm{km}$ asteroid near Mars $\left(18^{m}\right)$ & 95 \\
\hline
\end{tabular}

sit time (24 seconds, one viewing direction). Pluto, for example, moves on 20 mas. Completing the scan, one may calculate the image velocity and acceleration. Then one may pre-calculate positions of the object for future scans. Using the Pulkovo Observatory technique of "apparent motion parameters" (Kisselev, Bykov 1976) one may estimate a preliminary orbit of the object and then identify the object $2-4$ months later.

A simulation of Struve observations of two minor planets (451 Patientia and $1993 \mathrm{MF}$ ) shows rather big errors of $(O-C)$ values (up to $1^{\circ}$ ) for 4 months prediction time interval. But derivatives of the coordinates are in good correspondence with those calculated from the object's real orbit. This is very important for identification of unknown asteroids.

\section{Conclusions}

The Struve astrometric satellite will be an efficient instrument for investigation of the Solar System objects. The use of the Vlasov scheme of observations gives a uniform coverage of the ecliptic zone of the sky. A great number of new asteroids is expected to be discovered. The total number of moving objects to be detected by the Struve satellite is estimated as 50 to 80 thousands after 3 years of observations.

\section{References}

Kisselev, A.A., Bykov O.P. (1976) Determination of the elliptical orbit of a satellite with the use of parameters of its apparent motion, Astron.J., 53(4), 879-888.

Yershov, V.N., Kanayev, I.I. (1994) Development of the Russian Space Astrometric Satellite, Third International Workshop on Positional Astronomy and Celestial Mechanics, ed. A. López García, Cuenca. 